\title{
The Complete Evaporation Limit of Land Planets
}

\author{
Yuya Takao, Hidenori Genda, Miyuki Wakida and Yutaka Abe \\ Dept. of Earth and Planetary Science, The University of Tokyo, \\ 7-3-1 Hongo, Tokyo, Japan \\ email: takao@eps.s.u-tokyo.ac.jp
}

\begin{abstract}
Planets with very little amount of water on their surface, called "land planets", have wider habitable zones than that of Earth-like "aqua planets" (Abe et al. 2011). We investigated complete evaporation of surface liquid water for land planets using 1D energy balance model $(\mathrm{EBM})$. We found that complete evaporation occurs when the planetary flux at the dry edge, which is defined as the boundary between the dry zone and the wet zone, exceeds the critical radiation flux of water saturated atmosphere. We define "complete evaporation limit" as the minimum insolation for complete evaporation that a planet receives. This limit depends on latitude of the dry edge, efficiency of meridional heat transport, and atmospheric character that modify the value of the critical flux.
\end{abstract}

Keywords. stellar insolation; terrestrial planet - atmosphere

\section{Introduction}

Liquid water is thought to be essential for the origin and evolution of life on Earth. An Earth-sized planet globally covered with liquid water, called "aqua planet", will undergo a runaway water-vapor generated greenhouse effect if the planet is too close to the Sun, which leads to very high surface temperature and complete evaporation of liquid water. The critical value of the flux of the radiation that the planet can emit plays an essential role on runaway greenhouse effect. If the atmosphere is saturated with water-vapor, there appears an upper limit on the planetary radiation. Abe \& Matsui (1988) showed that the critical flux for an aqua planet was $305 \mathrm{~W} / \mathrm{m}^{2}$, which corresponds to $127 \%$ of the net insolation of present Earth (assuming 0.3 for planetary albedo). Kasting et al. (1988) calculated similar flux, which was around $309 \mathrm{~W} / \mathrm{m}^{2}$ (129\% of the net insolation of present Earth). However, for a planet with very little amount of water on its surface (land planet), the condition for appearance of the runaway greenhouse state would differ. Abe et al. (2011) showed that the inner edge of habitable zone for land planets is closer to the central star than that for aqua planets. They, using 3D general circulation model (GCM), have considered the climates of such dry planets, and have found that liquid water localizes near the poles while the low latitudes are dried up (if the planets have low obliquity). Such character enables the inner edge of habitable zone for land planets to be closer to the central star than that for aqua planets, because the atmosphere is not saturated and the tropics can emit radiation at rates above the critical flux for aqua planets. According to their numerical experiments, liquid water on a land planet is completely evaporated when the net insolation exceeds $415 \mathrm{~W} / \mathrm{m}^{2}$ (170\% of present Earth) for an Earth-sized planet with 1 bar air atmosphere.

Here, we will call such threshold the "complete evaporation limit". Abe et al. (2011) showed that the complete evaporation limit does not depend on the amount of water the planet initially has, if the planet is land planet. However, the complete evaporation limit 
was investigated for a certain limited conditions of a planet (1 bar air atmosphere, 0 obliquity and no transport of ground water). This limit also depends on the atmospheric pressure or composition, and transport of ground water.

Our goal is to understand the mechanisms that determine the complete evaporation limit, and to give rough prediction of the complete evaporation limit for any given planets. In this article we introduce the dependence of the complete evaporation limit on the degree of ground water localization and different amount of atmosphere.

\section{Models}

In order to understand the mechanisms determining the complete evaporation limit for land planets qualitatively, we use a meridional 1D energy balance model (EBM) used by North et al. (1981). This is a very simple model to estimate meridional temperature distribution of Earth, without atmospheric dynamics. Heat and water-vapor transport is given in forms of diffusion. Despite such important simplification, heat and water-vapor transport in the mid and high latitude is qualitatively correct, though it may not be correct in the low latitude where Hadley circulation dominates the process. Note that we are not concerned with precise values but mechanisms, and in order to investigate the climates quantitatively, we have to use much more precise model such as GCM, which is too complex to fully understand its behavior.

We have carried out numerical experiments to clarify the dependence of the complete evaporation limit on the degree of ground water localization. We supposed that the boundary between high-latitude wet area and low-latitude dry area, which we call the "dry edge", to be fixed at a certain latitude during calculation. We investigated the complete evaporation limit for various dry edge latitude, that is, various land fraction in the low latitudes. The latitude of the dry edge should be determined by a balance between water-vapor transport and ground water transport. Instead of solving ground water transport explicitly, we treated the dry edge latitude as a parameter to simplify the problem. Still, we can discuss how large ground water transport should be to maintain the given dry edge latitude, by checking corresponding water-vapor transport. An Earth-sized planet in circular orbit is considered. The obliquity of the planet is set to 0 . Planetary radiation flux is calculated with $1 \mathrm{D}$ gray radiative-convective equilibrium model used by Nakajima et al. (1992). This model has been designed for saturated atmosphere of aqua planets (supposing infinite water available), so we modified the model

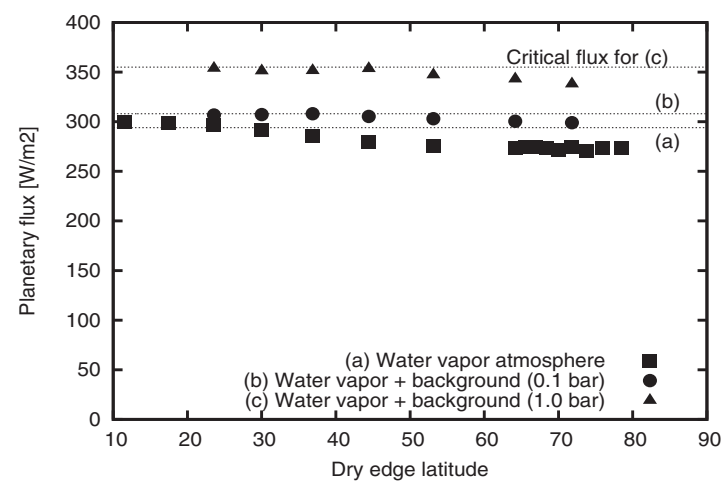

Figure 1. The planetary radiation at the dry edge, when complete evaporation is to occur if the stellar radiation increases by $1 \%$. Data points of squares show the results with atmosphere entirely composed of water-vapor. Considering water-vapor atmosphere with 0.1 bar and 1 bar of transparent component gives data points of circles and triangles, respectively. 


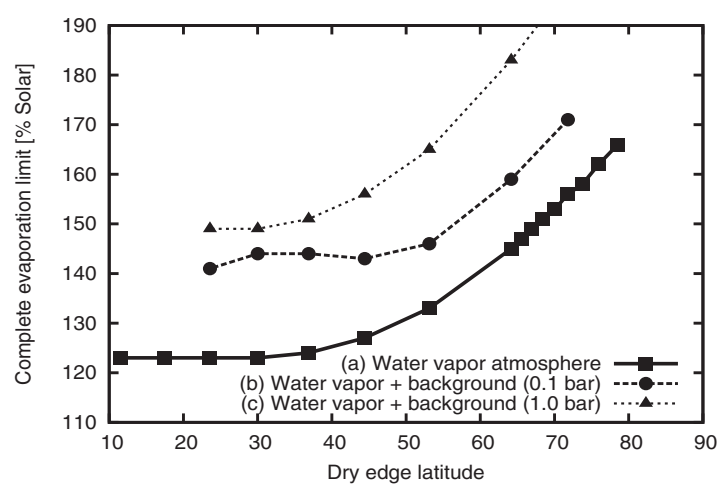

Figure 2. Response of the complete evaporation limit to various dry-edge latitude and the amount of atmosphere.

so that we can consider unsaturated atmosphere of land planets. Using this model, we obtained the relationships among surface temperature, amount of water-vapor, and planetary radiation flux.

\section{Mechanism of complete evaporation}

Figure 1 shows the planetary radiation at dry edges toward the stellar radiation just $1 \%$ smaller than that causes complete evaporation. The critical flux of each atmosphere is also shown in this figure. It is found, to zeroth order, that complete evaporation occurs when the planetary radiation at the dry edge reaches the critical flux. When the dry edge is at high latitude, the planetary radiation does not reach the critical flux. This may be related to the small planetary radiation from dry surface in low latitude, but we have to examine the mechanism more carefully. In addition, we have to investigate the effect of other atmospheric characters such as atmospheric component.

Figure 2 shows the dependence of the complete evaporation limit on the dry-edge latitude. If the dry edge is in low latitudes ( $<30$ degrees), the complete evaporation limit agrees with the complete evaporation limit of aqua planets (the critical flux). Otherwise, the higher the dry edge latitude exists, the larger the complete evaporation limit is. With larger amount of background atmospheric component, the complete evaporation limit gets larger because the critical flux of the atmosphere is raised. It is expected, however, that this tendency reaches a limit at some amount of transparent component, because it is known that there is a highest limit of the planetary radiation even if a vast amount (such as 100 bar) of transparent component exists (Nakajima et al. 1992).

\section{References}

Abe, Y., Abe-Ouchi, A., Sleep, N. H., \& Zahnle, K. J. 2011, Astrobiology, 11, 443

Abe, Y. \& Matsui, T. 1988, J. Atmos. Sci., 49, 3081

Kasting, J. F. 1988, Icarus, 74, 472

Nakajima, S., Hayashi, Y-Y., \& Abe, Y. 1992, J. Atmos. Sci., 49, 2256

North, G. R., Cahalan, R. F., \& Coakley, J. A. 1981, Rev. Geophys. Space Phys., 19, 91 